\title{
手性螺环骨架硼烷催化酮的不对称硅氢化反应
}

\author{
王桥天 $a, b$ 韩彩芳 $a, b$ 冯向青*,a,b 杜海峰*,,$b$ \\ ( ${ }^{a}$ 中国科学院化学研究所 北京国家分子科学研究中心 中国科学院分子识别与功能重点实验室 北京 100190) \\ $\left({ }^{b}\right.$ 中国科学院大学 北京 100049)
}

\begin{abstract}
摘要 受阻路易斯酸碱对(frustrated Lewis pairs, FLPs)是目前合成化学的前沿挑战性研究领域之一, 为非金属催化的氢 化和 Piers-type 硅氢化反应提供了非常有效的途径. 近年来, 相关研究取得了重要的研究进展, 但是相应的不对称反应 发展比较缓慢. 缺乏高效、高选择性的手性催化剂仍然是制约这一领域快速发展的重要因素. 手性螺环是配体设计中 的优势骨架. 基于前期所发展的联䒺骨架手性 FLP 催化剂及其在不对称催化氢化和硅氢化反应的应用, 设计并合成了 基于手性螺环骨架的手性双烯，通过与 $\mathrm{HB}\left(\mathrm{C}_{6} \mathrm{~F}_{5}\right)_{2}$ 的嗍氢化反应原位制备了新型手性嗍烷路易斯酸. 利用其与三叔丁基 膦形成的手性受阻路易斯酸碱对催化剂，成功地实现了简单酮的不对称 Piers-type 硅氢化反应，反应的对映选择性最高 可达 $90 \%$.
\end{abstract}

关键词＼cjkstart不对称催化; 不对称硅氢化; 受阻路易斯酸碱对; 手性螺二烯; 酮

\section{Chiral Spiro Dienes Derived Boranes for Asymmetric Hydrosilylation of Ketones}

\author{
Wang, Qiaotian ${ }^{a, b}$ \\ Han, Caifang ${ }^{a, b}$ \\ Feng, Xiangqing ${ }^{*, a, b}$ \\ $\mathrm{Du}$, Haifeng ${ }^{*, a, b}$ \\ ( ${ }^{a}$ Beijing National Laboratory for Molecular Sciences, CAS Key Laboratory of Molecular Recognition and Function, \\ Institute of Chemistry, Chinese Academy of Sciences, Beijing 100190) \\ ( ${ }^{b}$ University of Chinese Academy of Sciences, Beijing 100049)
}

\begin{abstract}
The chemistry of frustrated Lewis pairs (FLPs) is among the challenging frontiers of synthetic chemistry, which provides a powerful approach for metal-free catalytic hyrogenations and Piers-type hydrosilylations. In recent years, a significant progress has been made in this field. However, the deveopment of asymmetric reactions is still sluggish. The lacks of highly effective and enantioselective chiral FLP catalysts represent the key issue. $C_{2}$-symmetric 1,1 '-spirobiindane is one privileged framework in chiral ligands and catalysts. On the basis of chiral binaphthyl diene-derived frustrated Lewis pairs (FLPs) developed by our group, in this work, we designed and synthesized a novel class of chiral spiro dienes, which could further react with Piers' borane via the hydroboration reaction to generate chiral boranes in situ. With the combination of chiral borane and tri-tert-butylphosphine as an FLP catalyst, an asymmetric Piers-type hydrosilylation of simple ketones was successfully realized to give the desired secondary alcohols with up to $90 \%$ ee.
\end{abstract}

Keywords asymmetric catalysis; asymmetric hydrosilylation; frustrated Lewis pairs; chiral spiro dienes; ketones

\section{Introduction}

In 2006, Stephan and co-workers ${ }^{[1]}$ reported their seminal work on the reversible activation of $\mathrm{H}_{2}$ with sterically hindered Lewis acid and base pairs, which rapidly opened up a famous field as the chemistry of frustrated Lewis pairs (FLPs). In the past decade, the FLP-catalyzed metal-free hydrogenation and Piers-type hydrosilylation have achieved a great success. ${ }^{[2]}$ However, in contrast, the development of asymmetric reactions with chiral FLPs is still sluggish. ${ }^{[3]}$ Only a very few FLP catalysts can give satisfactory enantioselectivities. $^{[4,5]}$ The difficulty to access highly efficient chiral FLPs with suitable backbones and diverse structures seems to be the major obstacle in this field. At present, two protocols have been developed for the synthesis of chiral

\footnotetext{
* Corresponding authors. E-mail: fxq@iccas.ac.cn; haifengdu@iccas.ac.cn

Received March 30, 2019; revised April 24, 2019; published online May 10, 2019.

Dedicated to the 100th anniversary of the birth of Professor Ruyu Chen.

Project supported by the National Natural Science Foundation of China (No. 21825108).

国家自然科学基金(No. 21825108)资助项目.
} 
boron Lewis acids. One is hydroboration of chiral alkenes with Piers' borane $\mathrm{HB}\left(\mathrm{C}_{6} \mathrm{~F}_{5}\right)_{2},{ }^{[6]}$ and the other is substitution of boron chlorides with chiral organometallic reagents. ${ }^{[4]}$ As shown in Figure 1, the chiral frameworks of reported FLPs usually include bicycle, ${ }^{[5 b, 5 c, 5 e]} \quad C_{2}$-symmetric binaphthyl, ${ }^{[4 \mathrm{~b} \sim 4 \mathrm{f}]}$ and ferrocene. ${ }^{[5 \mathrm{~d}]}$ Recently, we have developed a novel strategy for the in situ synthesis of chiral boranes by the hydroboration of binaphthyl-based chiral dienes with $\mathrm{HB}\left(\mathrm{C}_{6} \mathrm{~F}_{5}\right)_{2} \cdot{ }^{[7]}$ Very recently, Wang et al. ${ }^{[8]}$ reported chiral $C_{2}$-symmetric bicyclic [3.3.0] and spiro-bicyclic diboranes. Generally, the chiral frameworks are very likely to influence the reactivity and enantioselectivity largely. Further developing chiral FLPs bearing novel backbones is therefore of great importance.

Spiro chirality has attracted considerable attention for the design of chiral ligands and catalysts, and numerous excellent chiral ligands and catalysts have already been successfully developed. ${ }^{[9]}$ It is noteworthy that $C_{2}$-symmetric 1,1'-spirobiindane $\mathbf{1}$ has proven to be one of privileged frameworks. ${ }^{[10]}$ On the basis of our previous work, we wish to further devote our efforts on the development of novel chiral dienes 2 bearing 1,1'-spirobiindane frameworks (Scheme 1). The corresponding chiral boranes 3 can be easily accessed via the in situ hydroboration with $\mathrm{HB}\left(\mathrm{C}_{6} \mathrm{~F}_{5}\right)_{2}$, which can be utilized in the FLP-catalyzed asymmetric reactions.

Piers-type hydrosilylation has received intensive atten- tion due to its synthetic and mechanistic interests. ${ }^{[1]}$ Despite some important advances, the asymmetric Piers-type hydrosilylation is far less developed in the past two decades. ${ }^{[2 i]}$ Especially for ketone substrates, very few examples can afford high enantioselectivities. ${ }^{[12]}$ In 2016, our group reported an asymmetric hydrosilylation of 1,2-dicarbonyl compounds using chiral diyne-derived alkenylborane and tricyclohexylphosphine to give up to $99 \% e e e^{[7 \mathrm{~d}]}$ Oestreich and co-workers ${ }^{[4 \mathrm{~d}]}$ employed the binaphthyl-based chiral borane to realize the asymmetric hydrosilylation of simple ketones with up to $99 \%$ ee. Herein, we report our preliminary results on the synthesis of chiral spiro boranes and their application in the asymmetric Piers-type hydrosilylation of simple ketones.

\section{Results and discussion}

The synthesis of chiral spiro dienes 2 with chiral dicarboxylic acid $\mathbf{4}^{[13]}$ as starting material was shown in Scheme 2. A Pd-catalyzed directed ortho- $\mathrm{C}-\mathrm{H}$ iodination of compound 4 gave diiodide 5 in $42 \%$ yield. ${ }^{[14]}$ Further treating diiodide $\mathbf{5}$ with thionyl dichloride in methanol furnished the corresponding ester 6 in $83 \%$ yield. Various aryl substituents can be easily incorporated by Suzuki coupling reactions to afford compounds 7 in moderate to good yields. A sequence DIBAL-H reduction and PCC oxidation gave dialdehydes 8 in reasonable yields. Followed by a
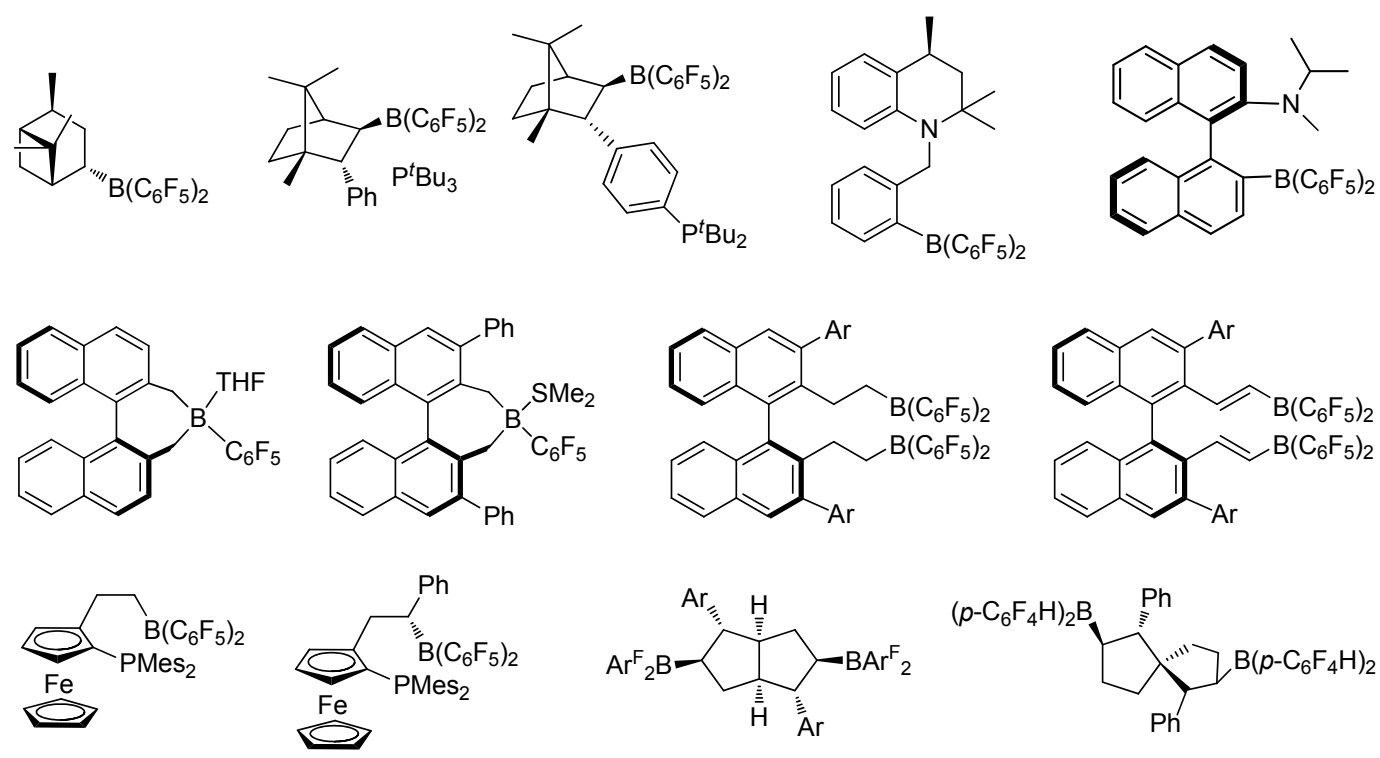

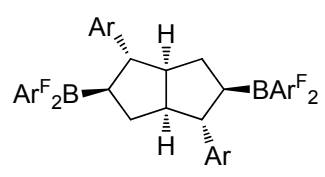

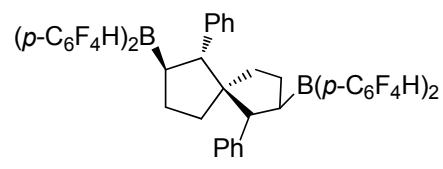

Figure 1 Representative chiral FLP catalysts

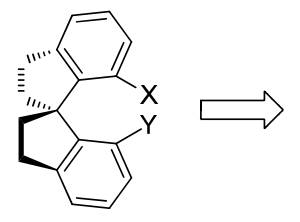

1

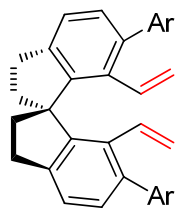

2

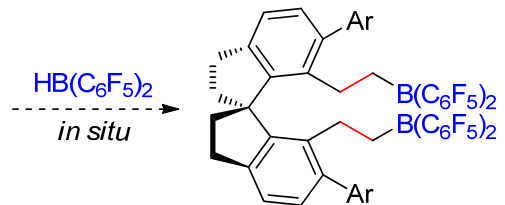

3

Scheme 1 The development of chiral spiro boranes 

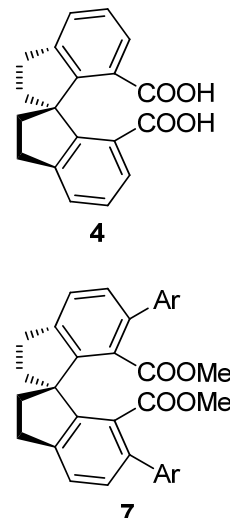

7

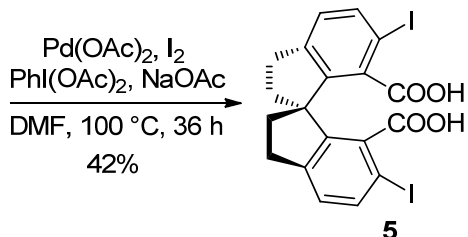

5

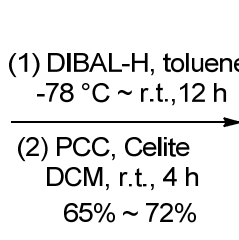

$65 \% \sim 72 \%$

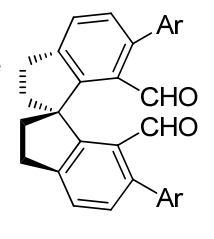

8

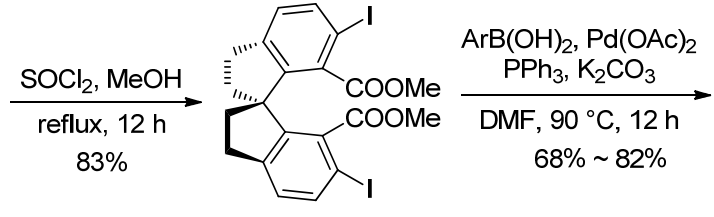

6

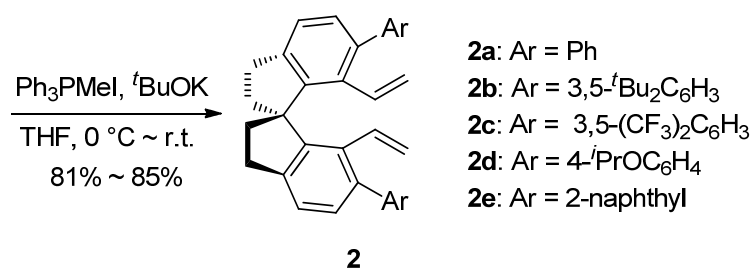

Scheme 2 Synthesis of the chiral spiro dienes 2

Wittig-reaction, chiral dienes $\mathbf{2 a} \sim \mathbf{2}$ e were obtained in $81 \% \sim 85 \%$ yields. A single crystal of chiral diene $2 \mathrm{e}$ was obtained, and its structure was confirmed by X-ray crystallographic analysis (Figure 2). ${ }^{[15]}$

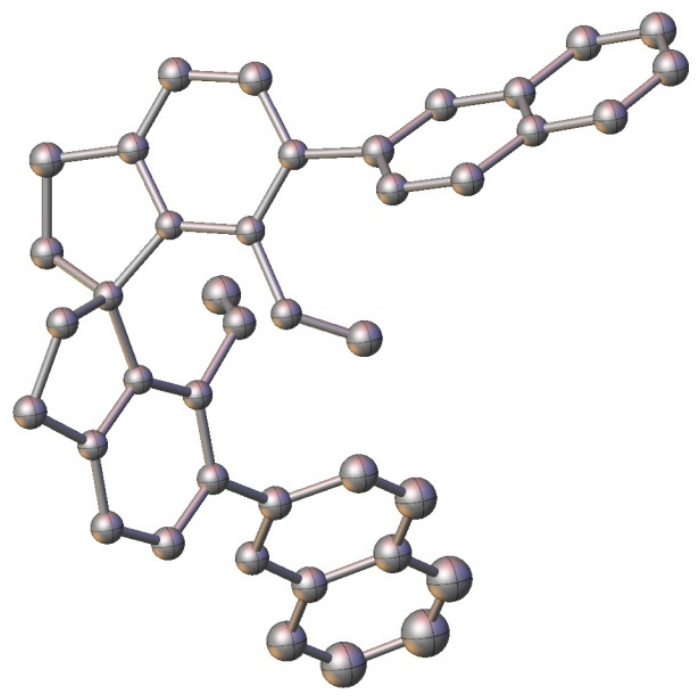

Figure 2 X-ray structure of $(S)$-2e

With novel chiral dienes $\mathbf{2}$ in hand, the asymmetric Piers-type hydrosilylation of acetophenone (9a) with $\mathrm{PhMe}_{2} \mathrm{SiH}$ was next investigated. As shown in Table 1, using chiral boranes generated in situ from dienes $\mathbf{2 a} \sim \mathbf{2 e}(5$ $\mathrm{mol} \%)$ and $\mathrm{HB}\left(\mathrm{C}_{6} \mathrm{~F}_{5}\right)_{2}(10 \mathrm{~mol} \%)$ in the presence of ${ }^{t} \mathrm{Bu}_{3} \mathrm{P}$ $(10 \mathrm{~mol} \%)$ as a Lewis base, all the reactions proceeded smoothly to give alcohol 10a in high conversions with low to moderate ee's (Entries $1 \sim 5$ ). Chiral diene $\mathbf{2 b}$ containing 3,5-di-tert-butylphenyl substituents gave the optimal enantioselectivity (Table 1, Entry 2). Lewis base components were found to have a large impact on the enantioselectivities (Table 1, Entries 2 vs 6 8). Without Lewis base, a much lower ee was obtained (Table 1, Entries 3 vs 9). Various silanes were subsequently studied, and $\mathrm{Ph}_{2} \mathrm{SiH}_{2}$ gave a promising 80\% ee (Table 1, Entries 2, 10 13). Reducing the catalyst loading from $10 \mathrm{~mol} \%$ to $5 \mathrm{~mol} \%$ only resulted in a slight loss of ee (Table 1, Entries 11 vs 14). Several solvents were also examined, and toluene proved to be a more suitable solvent (Table 1, Entries 14 17).
Various ketones 9a were subjected to the asymmetric hydrosilylation using chiral diene $2 \mathbf{b}(5 \mathrm{~mol} \%), \mathrm{HB}\left(\mathrm{C}_{6} \mathrm{~F}_{5}\right)_{2}$ (10 mol\%), and ${ }^{t} \mathrm{Bu}_{3} \mathrm{P}\left(10 \mathrm{~mol} \%\right.$ ), with $\mathrm{Ph}_{2} \mathrm{SiH}_{2}$ (1.5 equiv.) in toluene for $12 \mathrm{~h}$ followed by the deprotection of silyl group with tetrabutylammonium fluoride (TBAF). As shown in Scheme 3, all these reactions went well to furnish the desired secondary alcohols in $67 \% \sim 95 \%$ yields with 75\% 90\% ee's. 1-(Benzofuran-2-yl)ethan-1-one (9g) and 1-(naphthalen-1-yl)ethan-1-one (9h) were also effective substrates to afford the corresponding alcohols with $84 \%$ and $81 \%$ ee, respectively.

\section{Conclusions}

A variety of chiral dienes bearing $C_{2}$-symmetric $1,1^{\prime}$ spirobiindane framework were prepared in reasonable yields for the first time via a five-step synthesis with chiral dicarboxylic acid as starting material. The corresponding chiral spiro boranes were easily accessed by the in situ hydroboration with $\mathrm{HB}\left(\mathrm{C}_{6} \mathrm{~F}_{5}\right)_{2}$. Further application of these chiral boranes with ${ }^{t} \mathrm{Bu}_{3} \mathrm{P}$ as chiral FLP catalysts for the asymmetric Piers-type hydrosilylation of simple ketones to furnish the desired secondary alcohols in $67 \% \sim 95 \%$ yields with $75 \% \sim 90 \% e e$ 's. The unique spiro skeleton for this type of chiral boranes makes them potentially useful in other asymmetric reactions, and further efforts on this subject are still underway in our laboratory.

\section{Experimental section}

\subsection{General}

All air-sensitive compounds were handled under an atmosphere of argon or in a nitrogen-filled glovebox. All reagents and solvents were analytical grade materials purchased from commercial sources and used as received unless otherwise stated. Reactions were monitored by TLC (Qingdao Haiyang Chemical Co. Ltd. Silica gel $60 \mathrm{~F}_{254}$ ) and detected using an UV/Vis lamp (254 nm). Column chromatography was performed on a Qingdao Haiyang Chemical Co. Ltd. Silica gel 60 (200 300 mesh). 
Table 1 Optimization of reaction conditions for the asymmetric hydrosilylation ${ }^{a}$

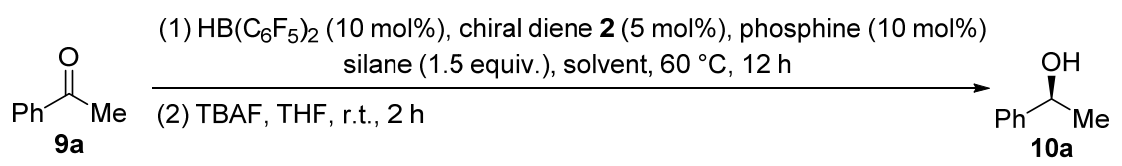

\begin{tabular}{|c|c|c|c|c|c|c|}
\hline Entry & Chiral diene 2 & Phosphine & Silane & Solvent & Conv. $/ \%$ & $e e / \%$ \\
\hline 1 & $2 a$ & ${ }^{t} \mathrm{Bu}_{3} \mathrm{P}$ & $\mathrm{PhMe}_{2} \mathrm{SiH}$ & Toluene & 95 & 40 \\
\hline 2 & $2 b$ & ${ }^{t} \mathrm{Bu}_{3} \mathrm{P}$ & $\mathrm{PhMe}_{2} \mathrm{SiH}$ & Toluene & $>99$ & 56 \\
\hline 3 & $2 \mathrm{c}$ & ${ }^{t} \mathrm{Bu}_{3} \mathrm{P}$ & $\mathrm{PhMe}_{2} \mathrm{SiH}$ & Toluene & 94 & 34 \\
\hline 4 & 2d & ${ }^{t} \mathrm{Bu}_{3} \mathrm{P}$ & $\mathrm{PhMe}_{2} \mathrm{SiH}$ & Toluene & 94 & 43 \\
\hline 5 & $2 \mathrm{e}$ & ${ }^{t} \mathrm{Bu}_{3} \mathrm{P}$ & $\mathrm{PhMe}_{2} \mathrm{SiH}$ & Toluene & 93 & 35 \\
\hline 6 & $2 b$ & $\mathrm{Cy}_{3} \mathrm{P}$ & $\mathrm{PhMe}_{2} \mathrm{SiH}$ & Toluene & 71 & 0 \\
\hline 7 & $2 b$ & $\mathrm{Mes}_{3} \mathrm{P}$ & $\mathrm{PhMe}_{2} \mathrm{SiH}$ & Toluene & 73 & 17 \\
\hline 8 & $2 b$ & $\left(\mathrm{C}_{6} \mathrm{~F}_{5}\right) \mathrm{Ph}_{2} \mathrm{P}$ & $\mathrm{PhMe}_{2} \mathrm{SiH}$ & Toluene & 96 & 0 \\
\hline 9 & $2 c$ & - & $\mathrm{PhMe}_{2} \mathrm{SiH}$ & Toluene & 91 & 23 \\
\hline 10 & $2 b$ & ${ }^{t} \mathrm{Bu}_{3} \mathrm{P}$ & $\mathrm{Et}_{3} \mathrm{SiH}$ & Toluene & 59 & 47 \\
\hline 11 & $2 b$ & ${ }^{t} \mathrm{Bu}_{3} \mathrm{P}$ & $\mathrm{Ph}_{2} \mathrm{SiH}_{2}$ & Toluene & $>99$ & 80 \\
\hline 12 & $2 b$ & ${ }^{t} \mathrm{Bu}_{3} \mathrm{P}$ & $\mathrm{PhSiH}_{3}$ & Toluene & $>99$ & 44 \\
\hline 13 & $2 b$ & ${ }^{t} \mathrm{Bu}_{3} \mathrm{P}$ & $\mathrm{Ph}_{3} \mathrm{SiH}$ & Toluene & 95 & 7 \\
\hline $14^{e}$ & $2 b$ & ${ }^{t} \mathrm{Bu}_{3} \mathrm{P}$ & $\mathrm{Ph}_{2} \mathrm{SiH}_{2}$ & Toluene & $>99$ & 79 \\
\hline $15^{e}$ & $2 b$ & ${ }^{t} \mathrm{Bu}_{3} \mathrm{P}$ & $\mathrm{Ph}_{2} \mathrm{SiH}_{2}$ & Hexane & $>99$ & 42 \\
\hline $16^{e}$ & $2 b$ & ${ }^{t} \mathrm{Bu}_{3} \mathrm{P}$ & $\mathrm{Ph}_{2} \mathrm{SiH}_{2}$ & THF & $>99$ & 0 \\
\hline $17^{e}$ & $2 \mathbf{b}$ & ${ }^{t} \mathrm{Bu}_{3} \mathrm{P}$ & $\mathrm{Ph}_{2} \mathrm{SiH}_{2}$ & DCM & 88 & 46 \\
\hline
\end{tabular}

${ }^{a}$ All reactions were carried out with acetophenone $(0.1 \mathrm{mmol}), \mathrm{HB}\left(\mathrm{C}_{6} \mathrm{~F}_{5}\right)_{2}(10 \mathrm{~mol} \%)$, chiral spiro diene $2(5 \mathrm{~mol} \%),{ }^{t} \mathrm{Bu}_{3} \mathrm{P}(10 \mathrm{~mol} \%)$, and silane (1.5 equiv.) in solvent $(1.0 \mathrm{~mL})$ at $60{ }^{\circ} \mathrm{C}$ for $12 \mathrm{~h}$, followed by removal the silyl group with TBAF at room temperature for $2 \mathrm{~h} .{ }^{b}$ Determined by crude ${ }^{1} \mathrm{H}$ NMR. ${ }^{c}$ Determined by chiral HPLC. ${ }^{e} \mathrm{HB}\left(\mathrm{C}_{6} \mathrm{~F}_{5}\right)_{2}(5 \mathrm{~mol} \%), \mathbf{2 b}(2.5 \mathrm{~mol} \%)$ and ${ }^{t} \mathrm{Bu}_{3} \mathrm{P}(5 \mathrm{~mol} \%)$ was used.

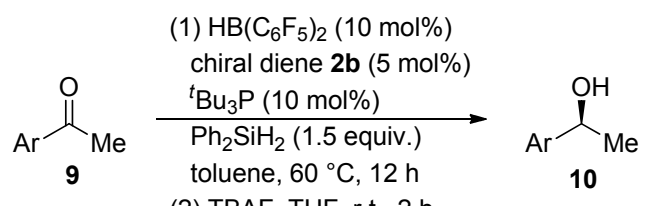

(2) TBAF, THF, r.t., $2 \mathrm{~h}$
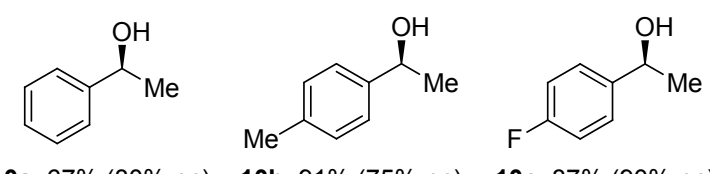

10a: $67 \%(80 \%$ ee $) \quad$ 10b: $91 \%(75 \%$ ee $) \quad$ 10c: $87 \%(90 \%$ ee $)$<smiles>C[C@H](O)c1ccc(Cl)cc1</smiles><smiles>C[C@H](O)c1ccc(Br)cc1</smiles><smiles>C[C@H](O)c1ccc(-c2ccccc2)cc1</smiles>

10d: $93 \%(90 \%$ ee) $\quad$ 10e: $93 \%(87 \%$ ee) $\quad$ 10f: $95 \%(86 \%$ ee $)$<smiles>C[C@H](O)c1cc2ccccc2o1</smiles><smiles>C[C@H](O)c1ccc2ccccc2c1</smiles>

10g: $86 \%$ (84\% ee) $\quad$ 10h: $95 \%(81 \%$ ee)

Scheme 3 Chiral FLP-catalyzed asymmetric hydrosilylation of ketones

${ }^{1} \mathrm{H}$ NMR and ${ }^{13} \mathrm{C}$ NMR spectra were recorded on a Bruker AV 400 at ambient temperature with $\mathrm{CDCl}_{3}$ and $\mathrm{C}_{6} \mathrm{D}_{6}$ as solvent and TMS as internal standard. Chemical shifts $(\delta)$ were referenced to the residual proton resonance of TMS (0) or to the carbon resonance of the $\mathrm{CDCl}_{3}$ (77.23), $\mathrm{C}_{6} \mathrm{D}_{6}$ (126.85). All solvents were purified by conventional methods, distilled before use. Commercially available rea- gents were used without further purification.

\subsection{Typical procedure for the synthesis of chiral diene $2 b$}

\subsubsection{Synthesis of compound $\mathbf{5}$}

Compound $(S)-4(0.2000 \mathrm{~g}, 0.66 \mathrm{mmol}), \mathrm{Pd}(\mathrm{OAc})_{2}$ $(0.0323 \mathrm{~g}, 0.132 \mathrm{mmol})$, iodobenzene diacetate $(0.5315 \mathrm{~g}$, $1.65 \mathrm{mmol})$ and $\mathrm{I}_{2}(0.4191 \mathrm{~g}, 1.65 \mathrm{mmol})$ were dissolved in $N, N$-dimethylformamide (DMF, $6.6 \mathrm{~mL}$ ) under argon. The tube was sealed with a cap and the reaction mixture was stirred at $100{ }^{\circ} \mathrm{C}$ for $36 \mathrm{~h}$. The reaction mixture was cooled to room temperature. After the solvent was evaporated to vacuum, saturated $\mathrm{Na}_{2} \mathrm{SO}_{3}(30 \mathrm{~mL})$ was added. The mixture was acidified with $2 \mathrm{~mol} / \mathrm{L} \mathrm{HCl}(10 \mathrm{~mL})$, extracted with dichloromethane (DCM, $10 \mathrm{~mL} \times 3)$ and dried over $\mathrm{Na}_{2} \mathrm{SO}_{4}$. The solvent was removed in a rotary evaporator and the residue $(S)$-6,6'-diiodo-2,2',3,3'-tetrahydro-1,1'spirobi[indene]-7,7'-dicarboxylic acid (5) (0.2060 g, 42\% yield) was used directly without other purification.

\subsubsection{Synthesis of compound 6}

To a $50 \mathrm{~mL}$ three-neck flash, $5(0.0900 \mathrm{~g}, 0.16 \mathrm{mmol})$ was dissolved in $\mathrm{SOCl}_{2}(2.1 \mathrm{~mL})$ under argon. The reaction mixture was refluxed at $90{ }^{\circ} \mathrm{C}$ for $8 \mathrm{~h}$, and then solvent was evaporated directly. $\mathrm{MeOH}(20 \mathrm{~mL})$ was added, and it was refluxed at $90{ }^{\circ} \mathrm{C}$ for $12 \mathrm{~h}$. The reaction mixture was cooled to room temperature. The solvent was filtered, residue was collected to afford target product 6 as a white solid $(0.0764$ g, $82 \%$ yield). m.p. $151 \sim 154{ }^{\circ} \mathrm{C} ;{ }^{1} \mathrm{H}$ NMR (400 MHz, $\left.\mathrm{CDCl}_{3}\right) \delta: 7.68(\mathrm{~d}, J=8.0 \mathrm{~Hz}, 2 \mathrm{H}), 7.01(\mathrm{~d}, J=8.0 \mathrm{~Hz}, 2 \mathrm{H})$, $3.25(\mathrm{~s}, 6 \mathrm{H}), 3.04 \sim 2.89(\mathrm{~m}, 4 \mathrm{H}), 2.67 \sim 2.56(\mathrm{~m}, 2 \mathrm{H})$, 
$2.27 \sim 2.16(\mathrm{~m}, 2 \mathrm{H}) ;{ }^{13} \mathrm{C} \mathrm{NMR}\left(100 \mathrm{MHz}, \mathrm{CDCl}_{3}\right) \delta: 167.8$, $147.3,145.3,138.1,136.4,127.2,90.4,62.7,51.4,39.8$, 30.3; HRMS (APCI) calcd for $\mathrm{C}_{21} \mathrm{H}_{19} \mathrm{O}_{4} \mathrm{I}_{2}[\mathrm{M}+\mathrm{H}]^{+}$ 588.9367, found 588.9360.

\subsubsection{Synthesis of compound 7}

To a $50 \mathrm{~mL}$ three-neck flash, $6(0.5880 \mathrm{~g}, 1.0 \mathrm{mmol})$, (3,5-di-tert-butylphenyl)boronic acid $(0.7020 \mathrm{~g}, 3.0 \mathrm{mmol})$, $\mathrm{Pd}(\mathrm{OAc})_{2}(0.0112 \mathrm{~g}, 0.05 \mathrm{mmol}), \mathrm{PPh}_{3}(0.0393 \mathrm{~g}, 0.15$ $\mathrm{mmol}), \mathrm{K}_{2} \mathrm{CO}_{3}(0.4140 \mathrm{~g}, 3.0 \mathrm{mmol})$ were dissolved in DMF $(25 \mathrm{~mL})$ under argon. The reaction mixture was stirred at $90{ }^{\circ} \mathrm{C}$ for $12 \mathrm{~h}$. The reaction mixture was cooled to room temperature, the mixture was poured into saturated $\mathrm{NH}_{4} \mathrm{Cl}$ (aq.) and extracted with $\mathrm{Et}_{2} \mathrm{O}(20 \mathrm{~mL} \times 2)$. The organic layers were combined, dried over $\mathrm{Na}_{2} \mathrm{SO}_{4}$, filtered. After removal of solvents, the residue was purified by flash column chromatography on silica gel to afford dimethyl (S)-6,6'-bis(3,5-di-tert-butylphenyl)-2,2',3,3'-tetrahydro1,1'-spirobi[indene]-7,7'-dicarboxylate (7b) (0.6070 g, 82\% yield) as a white solid. m.p. $227 \sim 229{ }^{\circ} \mathrm{C} ;{ }^{1} \mathrm{H}$ NMR $(400$ $\left.\mathrm{MHz}, \mathrm{CDCl}_{3}\right) \delta: 7.35(\mathrm{~s}, 1 \mathrm{H}), 7.33(\mathrm{~s}, 1 \mathrm{H}), 7.32 \sim 7.30(\mathrm{~m}$, $2 \mathrm{H}), 7.25(\mathrm{~s}, 1 \mathrm{H}), 7.23(\mathrm{~s}, 1 \mathrm{H}), 7.11(\mathrm{~d}, J=2.5 \mathrm{~Hz}, 4 \mathrm{H})$, $3.19 \sim 3.03(\mathrm{~m}, 4 \mathrm{H}), 2.88(\mathrm{~s}, 6 \mathrm{H}), 2.87 \sim 2.78(\mathrm{~m}, 2 \mathrm{H})$, $2.43 \sim 2.35(\mathrm{~m}, 2 \mathrm{H}), 1.29(\mathrm{~s}, 36 \mathrm{H}) ;{ }^{13} \mathrm{C} \mathrm{NMR}(125 \mathrm{MHz}$, $\left.\mathrm{CDCl}_{3}\right) \delta: 169.1,150.3,146.3,144.0,140.5,140.4,129.6$, $128.6,125.2,122.7,120.6,62.2,50.7,39.9,34.8,31.5$, 30.4; HRMS (APCI) calcd for $\mathrm{C}_{47} \mathrm{H}_{53} \mathrm{O}_{2}[\mathrm{M}+\mathrm{H}]^{+}$ 649.4040 , found 649.4030 .

\subsubsection{Synthesis of compound 8}

To a $50 \mathrm{~mL}$ three-neck flash, $7 \mathbf{b}(0.4000 \mathrm{~g}, 0.56 \mathrm{mmol})$ was dissolved in toluene $(10 \mathrm{~mL})$ under argon. The reaction mixture was cooled down to $-78{ }^{\circ} \mathrm{C}$, DIBAL-H $(1.5$ $\mathrm{mol} / \mathrm{L}, 1.5 \mathrm{~mL}, 1.7 \mathrm{mmol}$ ) was dropwise added and then stirred at $-78{ }^{\circ} \mathrm{C}$ for $3 \mathrm{~h}$. After that mixture was warmed up to room temperature and further stirred overnight. The mixture was poured into saturated $\mathrm{NH}_{4} \mathrm{Cl}$ (aq.) and extracted with $\mathrm{Et}_{2} \mathrm{O}(20 \mathrm{~mL} \times 2)$. The organic layers were combined, dried over $\mathrm{Na}_{2} \mathrm{SO}_{4}$, and filtered. Solvent was removed under reduced pressure to afford a mixture of dialcohols $(0.3930 \mathrm{~g})$. The mixture was dissolved in DCM $(25 \mathrm{~mL})$ in a $50 \mathrm{~mL}$ three-neck flash under argon, then PCC $(1.3000 \mathrm{~g}, 6 \mathrm{mmol})$, Celite $(0.3000 \mathrm{~g})$ were added subsequently. The reaction mixture was stirred at room temperature for $4 \mathrm{~h}$. The resulting suspension solution was filtered through a short pad of Celite, and the solid was washed with $\mathrm{CH}_{2} \mathrm{Cl}_{2}(5.0 \mathrm{~mL} \times 2)$. After removal of the solvents, the residue was purified by flash chromatography on silica gel to afford $(S)$-6,6'-bis(3,5-di-tert-butylphenyl)-2,2',3,3'tetrahydro-1,1'-spirobi[indene]-7,7'-dicarbaldehyde $\quad(\mathbf{8 b})$ $(0.2450 \mathrm{~g}, 67 \%$ yield $)$ as a white solid. m.p. $121 \sim 125^{\circ} \mathrm{C}$; ${ }^{1} \mathrm{H}$ NMR (400 MHz, $\left.\mathrm{CDCl}_{3}\right) \delta: 9.50(\mathrm{~s}, 2 \mathrm{H}), 7.53(\mathrm{~d}, J=8.0$ $\mathrm{Hz}, 2 \mathrm{H}), 7.39 \sim 7.36(\mathrm{~m}, 2 \mathrm{H}), 7.31$ (d, $J=7.6 \mathrm{~Hz}, 2 \mathrm{H}), 7.08$ $(\mathrm{d}, J=1.6 \mathrm{~Hz}, 4 \mathrm{H}), 3.31 \sim 3.09(\mathrm{~m}, 4 \mathrm{H}), 2.79 \sim 2.66(\mathrm{~m}$, 2H), $2.50 \sim 2.40(\mathrm{~m}, 2 \mathrm{H}), 1.29(\mathrm{~s}, 36 \mathrm{H}) ;{ }^{13} \mathrm{C}$ NMR $(125$ $\left.\mathrm{MHz}, \mathrm{CDCl}_{3}\right) \delta: 193.7,150.5,149.7,145.3,144.8,138.2$, $129.7,129.4,128.3,124.7,121.4,63.3,39.5,34.8,31.4$, 30.7; HRMS (APCI) calcd for $\mathrm{C}_{47} \mathrm{H}_{57} \mathrm{O}_{2}[\mathrm{M}+\mathrm{H}]^{+}$
653.4353 , found 653.4338 .

\subsubsection{Synthesis of compound 2}

To a $10 \mathrm{~mL}$ Schlenk tube, $\mathrm{PPh}_{3} \mathrm{MeI}(1.1000 \mathrm{~g}, 2.7 \mathrm{mmol})$ and THF $(5.0 \mathrm{~mL})$ was cooled to $0{ }^{\circ} \mathrm{C}$, then ${ }^{t} \mathrm{BuOK}(0.3020$ $\mathrm{g}, 2.7 \mathrm{mmol})$ was added. After that, the solution of $\mathbf{8 b}$ $(0.1740 \mathrm{~g}, 0.27 \mathrm{mmol})$ in THF $(1.0 \mathrm{~mL})$ was added subsequently. The reaction mixture was stirred for $20 \mathrm{~min}$ and quenched with water, extracted with $\mathrm{Et}_{2} \mathrm{O}(5 \mathrm{~mL} \times 2)$. The organic phase was dried over $\mathrm{Na}_{2} \mathrm{SO}_{4}$, filtered. After removal of the solvent, the residue was purified by flash column chromatography $[V$ (hexanes) $: V(\mathrm{DCM})=100: 1]$ to give chiral diene $\mathbf{2 b}$ as a white solid $(0.1410 \mathrm{~g}, 81 \%$ yield). Chiral dienes $\mathbf{2 a}, \mathbf{2} \mathbf{c} \sim \mathbf{2 e}$ were synthesized by this method.

(S)-6,6'-Diphenyl-7,7'-divinyl-2,2',3,3'-tetrahydro-1,1'spirobi[indene] (2a): White solid, m.p. $100 \sim 103{ }^{\circ} \mathrm{C}$; $[\alpha]_{\mathrm{D}}^{27}$ - 457.2 (c 0.5, $\left.\mathrm{CH}_{2} \mathrm{Cl}_{2}\right) ;{ }^{1} \mathrm{H}$ NMR $\left(500 \mathrm{MHz}, \mathrm{C}_{6} \mathrm{D}_{6}\right) \delta: 7.33$ $(\mathrm{d}, J=7.5 \mathrm{~Hz}, 4 \mathrm{H}), 7.20 \sim 7.12(\mathrm{~m}, 6 \mathrm{H}), 7.09 \sim 7.01(\mathrm{~m}$, $4 \mathrm{H}), 6.52(\mathrm{dd}, J=17.5,11.5 \mathrm{~Hz}, 2 \mathrm{H}), 4.79(\mathrm{dd}, J=19.0$, $11.5 \mathrm{~Hz}, 4 \mathrm{H}), 2.93 \sim 2.72(\mathrm{~m}, 4 \mathrm{H}), 2.33(\mathrm{q}, J=10.5 \mathrm{~Hz}$, $2 \mathrm{H}), 2.12 \sim 2.03(\mathrm{~m}, 2 \mathrm{H}) ;{ }^{13} \mathrm{C} \mathrm{NMR}\left(125 \mathrm{MHz}, \mathrm{C}_{6} \mathrm{D}_{6}\right) \delta$ : $146.9,143.1,142.4,140.2,133.9,133.3,130.3,130.1$, $127.9,126.1,123.5,120.6,62.4,37.8,30.3$; IR (film) $v$ : 3360, 2929, 2848, 1460, 919, $713 \mathrm{~cm}^{-1}$; HRMS (APCI) calcd for $\mathrm{C}_{33} \mathrm{H}_{31}[\mathrm{M}+\mathrm{H}]^{+}$425.2264, found 425.2260.

(S)-6,6'-Bis(3,5-di-tert-butylphenyl)-7,7'-divinyl2,2',3,3'-tetrahydro-1,1'-spirobi[indene] (2b): White solid, m.p. $121 \sim 123{ }^{\circ} \mathrm{C} ;[\alpha]_{\mathrm{D}}^{27}-278.7\left(c \quad 0.53, \mathrm{CH}_{2} \mathrm{Cl}_{2}\right) ;{ }^{1} \mathrm{H}$ NMR $\left(300 \mathrm{MHz}, \mathrm{CDCl}_{3}\right) \delta: 7.29 \sim 7.24(\mathrm{t}, J=1.6 \mathrm{~Hz}, 2 \mathrm{H})$, 7.23 (d, $J=2.4 \mathrm{~Hz}, 1 \mathrm{H}), 7.20$ (d, $J=2.4 \mathrm{~Hz}, 3 \mathrm{H}), 7.11$ (d, $J=1.8 \mathrm{~Hz}, 4 \mathrm{H}), 6.20(\mathrm{dd}, J=17.7,11.4 \mathrm{~Hz}, 2 \mathrm{H}), 4.76(\mathrm{dd}$, $J=11.4,1.8 \mathrm{~Hz}, 2 \mathrm{H}), 4.49(\mathrm{dd}, J=17.7,1.8 \mathrm{~Hz}, 2 \mathrm{H}), 3.14 \sim$ $2.88(\mathrm{~m}, 4 \mathrm{H}), 2.37 \sim 2.23(\mathrm{~m}, 4 \mathrm{H}), 1.29(\mathrm{~s}, 36 \mathrm{H}) ;{ }^{13} \mathrm{C} \mathrm{NMR}$ $\left(125 \mathrm{MHz}, \mathrm{C}_{6} \mathrm{D}_{6}\right) \delta: 150.0,147.0,142.3,142.2,141.3$, $134.1,133.6,130.3,124.9,123.4,120.2,119.6,62.5,37.7$, 34.6, 31.4, 30.3; IR (film): 3055, 2961, 2865, 1595, 1249, $917,746 \mathrm{~cm}^{-1}$; HRMS (APCI) calcd for $\mathrm{C}_{49} \mathrm{H}_{61}[\mathrm{M}+\mathrm{H}]^{+}$ 649.4768 , found 649.4757 .

(S)-6,6'-Bis(3,5-bis(trifluoromethyl)phenyl)-7,7'-divinyl2,2',3,3'-tetrahydro-1,1'-spirobi[indene] (2c): White solid, m.p. $127 \sim 129{ }^{\circ} \mathrm{C} ;[\alpha]_{\mathrm{D}}^{27}-283.8\left(c \quad 0.39, \mathrm{CH}_{2} \mathrm{Cl}_{2}\right) ;{ }^{1} \mathrm{H}$ NMR (500 MHz, $\left.\mathrm{C}_{6} \mathrm{D}_{6}\right) \delta: 7.66(\mathrm{~s}, 2 \mathrm{H}), 7.58(\mathrm{~s}, 4 \mathrm{H}), 6.99$ $(\mathrm{d}, J=8.0 \mathrm{~Hz}, 2 \mathrm{H}), 6.78$ (d, $J=7.5 \mathrm{~Hz}, 2 \mathrm{H}), 6.19$ (dd, $J=$ $17.5,11.0 \mathrm{~Hz}, 2 \mathrm{H}), 4.58(\mathrm{~d}, J=10.0 \mathrm{~Hz}, 2 \mathrm{H}), 4.36(\mathrm{~d}, J=$ $16.5, \mathrm{~Hz}, 2 \mathrm{H}), 2.85 \sim 2.66(\mathrm{~m}, 4 \mathrm{H}), 2.19 \sim 2.08(\mathrm{~m}, 2 \mathrm{H})$, $2.05 \sim 1.97(\mathrm{~m}, 2 \mathrm{H}) ;{ }^{13} \mathrm{C}$ NMR $\left(125 \mathrm{MHz}, \mathrm{C}_{6} \mathrm{D}_{6}\right) \delta: 146.8$, $144.7,143.8,136.7,133.8,132.6,131.2$ (q, $J=32.9 \mathrm{~Hz})$, $130.3,130.15,124.0,123.6$ (d, $J=271.0 \mathrm{~Hz}), 121.9,119.9$, 62.0, 37.5, 30.3; ${ }^{19} \mathrm{~F}$ NMR (376 MHz, $\left.\mathrm{CDCl}_{3}\right) \delta$ : -62.62 ; IR (film) $v:$ 2920, 2848, 1644, 1379, 1278, 1133, 740 $\mathrm{cm}^{-1}$; HRMS (APCI) calcd for $\mathrm{C}_{37} \mathrm{H}_{23} \mathrm{~F}_{2}(\mathrm{M}-\mathrm{H})$ 695.1614; Found: 695.1620 .

$(S)-6,6$ '-Bis(4-isopropoxyphenyl)-7,7'-divinyl-2,2',3,3'tetrahydro-1,1'-spirobi[indene] (2d): White solid, m.p. $123 \sim 125{ }^{\circ} \mathrm{C} ;[\alpha]_{\mathrm{D}}^{27}-390.1\left(c \quad 0.78, \mathrm{CH}_{2} \mathrm{Cl}_{2}\right) ;{ }^{1} \mathrm{H} \mathrm{NMR}$ $\left(500 \mathrm{MHz}, \mathrm{C}_{6} \mathrm{D}_{6}\right) \delta: 7.31 \sim 7.27(\mathrm{~m}, 4 \mathrm{H}), 7.25$ (d, $J=8.0$ 
$\mathrm{Hz}, 2 \mathrm{H}), 7.08(\mathrm{~d}, J=7.5 \mathrm{~Hz}, 2 \mathrm{H}), 6.87 \sim 6.80(\mathrm{~m}, 4 \mathrm{H})$, $6.63 \sim 6.54(\mathrm{~m}, 2 \mathrm{H}), 4.88(\mathrm{dq}, J=14.5,1.5 \mathrm{~Hz}, 4 \mathrm{H}), 4.24 \sim$ $4.14(\mathrm{~m}, 2 \mathrm{H}), 2.94 \sim 2.75(\mathrm{~m}, 4 \mathrm{H}), 2.43 \sim 2.32(\mathrm{~m}, 2 \mathrm{H})$, $2.16 \sim 2.07(\mathrm{~m}, 2 \mathrm{H}), 1.11(\mathrm{~d}, J=6.0 \mathrm{~Hz}, 12 \mathrm{H}) ;{ }^{13} \mathrm{C} \mathrm{NMR}$ $\left(125 \mathrm{MHz}, \mathrm{C}_{6} \mathrm{D}_{6}\right) \delta: 156.0,146.1,141.2,139.1,134.3$, 133.1, 132.8, 130.3, 129.5, 122.7, 119.5, 114.4, 68.2, 61.6, 37.0, 29.5, 20.9(2), 20.9(0); IR (film) v: 3361, 2975, 2934, 2848, 1512, 1240, 1119, $819 \mathrm{~cm}^{-1}$; HRMS (APCI) calcd for $\mathrm{C}_{39} \mathrm{H}_{41} \mathrm{O}_{2}[\mathrm{M}+\mathrm{H}]^{+}$541.3101, found 541.3097.

(S)-6,6'-Di(naphthalen-2-yl)-7,7'-divinyl-2,2',3,3'-tetrahydro-1,1'-spirobi[indene] (2e): White solid, m.p. $221 \sim$ $223{ }^{\circ} \mathrm{C} ;[\alpha]_{\mathrm{D}}^{27}-547.8\left(\mathrm{c} 0.54, \mathrm{CH}_{2} \mathrm{Cl}_{2}\right) ;{ }^{1} \mathrm{H}$ NMR $(400$ $\left.\mathrm{MHz}, \mathrm{CDCl}_{3}\right) \delta: 7.77 \sim 7.68(\mathrm{~m}, 6 \mathrm{H}), 7.66(\mathrm{~d}, J=8.4 \mathrm{~Hz}$, 2H), $7.41 \sim 7.33(\mathrm{~m}, 4 \mathrm{H}), 7.28(\mathrm{~d}, J=8.4 \mathrm{~Hz}, 2 \mathrm{H}), 7.21 \sim$ $7.13(\mathrm{~m}, 4 \mathrm{H}), 6.21(\mathrm{dd}, J=17.6,11.6 \mathrm{~Hz}, 2 \mathrm{H}), 4.74(\mathrm{~d}, J=$ $12.0,2 \mathrm{H}), 4.53(\mathrm{~d}, \quad J=17.6 \mathrm{~Hz}, 2 \mathrm{H}), 3.08 \sim 2.86(\mathrm{~m}, 4 \mathrm{H})$, $2.33 \sim 2.18(\mathrm{~m}, 4 \mathrm{H}) ;{ }^{13} \mathrm{C} \mathrm{NMR}\left(100 \mathrm{MHz}, \mathrm{CDCl}_{3}\right) \delta: 146.9$, $142.8,140.7,139.6,134.1,133.5,133.2,132.0,130.4$, $129.2,128.2,127.9,127.6,126.9,125.9,125.6,123.5$, 121.2, 62.3, 37.9, 30.5; IR (film) v: 3054, 2939, 1265, 742 $\mathrm{cm}^{-1}$; HRMS (APCI) calcd for $\mathrm{C}_{41} \mathrm{H}_{33}[\mathrm{M}+\mathrm{H}]^{+}$525.2577, found 525.2573 .

4.3 Typical procedure for the metal-free catalytic asymmetric hydrosilylation of ketone

To a $15 \mathrm{~mL}$ sealed tube was added $\mathrm{HB}\left(\mathrm{C}_{6} \mathrm{~F}_{5}\right)_{2}(0.0107 \mathrm{~g}$, $0.03 \mathrm{mmol})$, chiral diene $2 \mathbf{b}(0.0097 \mathrm{~g}, 0.015 \mathrm{mmol})$, and dry toluene $(3.0 \mathrm{~mL})$ in a nitrogen atmosphere glovebox. The resulting mixture was stirred for $5 \mathrm{~min}$ at room temperature followed by addition of $\mathrm{Ph}_{2} \mathrm{SiH}_{2}(0.0830 \mathrm{~g}, 0.45$ $\mathrm{mmol}$ ), $\mathrm{P}^{t} \mathrm{Bu}_{3}(10 \mathrm{wt} \%$ in $n$-pentane, $0.0607 \mathrm{~g}, 0.03 \mathrm{mmol}$ ), and ketone 9a $(0.0360 \mathrm{~g}, 0.3 \mathrm{mmol})$. The reaction mixture was stirred at $60{ }^{\circ} \mathrm{C}$ for $12 \mathrm{~h}$. The reaction mixture was cooled to room temperature and TBAF $(0.3 \mathrm{mmol}, 0.3 \mathrm{~mL}$, $1.0 \mathrm{~mol} / \mathrm{L}$ in THF) was added and stirred at room temperature for $2 \mathrm{~h}$. The resulting solution was added water (15 $\mathrm{mL})$ and extracted with $\mathrm{CH}_{2} \mathrm{Cl}_{2}(10 \mathrm{~mL} \times 3)$. The resulting residue was purified by column chromatography on silica gel (petroleum ether/ethyl acetate, $V / V=50 / 1$ ) to afford the desired $(S)$-10a as a colorless oil $(0.0246 \mathrm{~g}, 67 \%$ yield, $80 \%$ ee). $\mathbf{1 0 b} \sim 10 \mathrm{~h}$ were obtained in a similar manner.

(S)-1-Phenylethan-1-ol (10a): Colorless oil, $0.0246 \mathrm{~g}$, $67 \%$ yield, $80 \%$ ee. $[\alpha]_{\mathrm{D}}^{27}-29.4\left(\mathrm{c} 0.76, \mathrm{CHCl}_{3}\right)$ [lit. ${ }^{[16 \mathrm{a}]}$ $[\alpha]_{\mathrm{D}}^{24}+42.92\left(c 1.04, \mathrm{CHCl}_{3}\right)(96 \%$ ee for $R$-isomer $\left.)\right] ;{ }^{1} \mathrm{H}$ NMR $\left(500 \mathrm{MHz}, \mathrm{CDCl}_{3}\right) \delta: 7.37 \sim 7.30(\mathrm{~m}, 4 \mathrm{H}), 7.28 \sim$ $7.22(\mathrm{~m}, 1 \mathrm{H}), 4.84(\mathrm{q}, J=6.5 \mathrm{~Hz}, 1 \mathrm{H}), 2.22(\mathrm{~s}, 1 \mathrm{H}), 1.46(\mathrm{~d}$, $J=6.5 \mathrm{~Hz}, 3 \mathrm{H}) ;{ }^{13} \mathrm{C} \mathrm{NMR}\left(125 \mathrm{MHz}, \mathrm{CDCl}_{3}\right) \delta: 145.8$, $128.5,127.4,125.4,70.3,25.1$. Enantiomeric excess was determined by HPLC with a Chiralcel OD-H column $\left[V(\right.$ hexane $\left.) / V\left({ }^{i} \mathrm{PrOH}\right)=95 / 5,1.0 \mathrm{~mL} / \mathrm{min}, 210 \mathrm{~nm}\right]$, minor enantiomer $t_{\mathrm{r}}=8.71 \mathrm{~min}$, major enantiomer $t_{\mathrm{r}}=10.01 \mathrm{~min}$.

(S)-1-(p-Tolyl)ethan-1-ol (10b): Colorless oil, $0.0370 \mathrm{~g}$, $91 \%$ yield, $75 \%$ ee. $[\alpha]_{\mathrm{D}}^{27}-39.2\left(c 1.04, \mathrm{CH}_{2} \mathrm{Cl}_{2}\right)\left[\right.$ lit. ${ }^{[16 \mathrm{~b}]}$ $[\alpha]_{\mathrm{D}}^{26}+55.2\left(c 0.50, \mathrm{CH}_{2} \mathrm{Cl}_{2}\right)(99.7 \%$ ee for $R$-isomer $\left.)\right] ;{ }^{1} \mathrm{H}$ NMR (400 MHz, $\left.\mathrm{CDCl}_{3}\right) \delta: 7.22(\mathrm{~d}, J=8.0 \mathrm{~Hz}, 2 \mathrm{H}), 7.12$ (d, $J=8.0 \mathrm{~Hz}, 2 \mathrm{H}), 4.79$ (q, $J=6.4 \mathrm{~Hz}, 1 \mathrm{H}), 2.32(\mathrm{~s}, 3 \mathrm{H})$, 2.22 (br s, $1 \mathrm{H}), 1.43$ (d, $J=6.4 \mathrm{~Hz}, 3 \mathrm{H}) ;{ }^{13} \mathrm{C}$ NMR $(100$
$\left.\mathrm{MHz}, \mathrm{CDCl}_{3}\right) \delta: 143.0,137.1,129.2,125.4,70.2,25.1$, 21.1; Enantiomeric excess was determined by HPLC with a Chiralcel OJ-H column $\left[V(\right.$ hexane $) / V\left({ }^{i} \mathrm{PrOH}\right)=95 / 5,1.0$ $\mathrm{mL} / \mathrm{min}, 220 \mathrm{~nm}$ ], major enantiomer $t_{\mathrm{r}}=12.08 \mathrm{~min}$, minor enantiomer $t_{\mathrm{r}}=13.22 \mathrm{~min}$.

(S)-1-(4-Fluorophenyl)ethan-1-ol (10c): Colorless oil, $0.0360 \mathrm{~g}, 87 \%$ yield, $90 \%$ ee. $[\alpha]_{\mathrm{D}}^{27}-52.8\left(\mathrm{c} 1.20, \mathrm{CH}_{2} \mathrm{Cl}_{2}\right)$ [lit. ${ }^{[16 \mathrm{~b}]}[\alpha]_{\mathrm{D}}^{20}+49.0\left(c \quad 0.20, \mathrm{CH}_{2} \mathrm{Cl}_{2}\right) \quad(99.9 \%$ ee for $R$-isomer)]; ${ }^{1} \mathrm{H}$ NMR (400 MHz, $\left.\mathrm{CDCl}_{3}\right) \delta: 7.50 \sim 7.38(\mathrm{~m}$, $2 \mathrm{H}), 7.17 \sim 7.06(\mathrm{~m}, 2 \mathrm{H}), 4.98(\mathrm{q}, J=6.4 \mathrm{~Hz}, 1 \mathrm{H}), 1.87(\mathrm{br}$ $\mathrm{s}, 1 \mathrm{H}), 1.56(\mathrm{~d}, J=6.4 \mathrm{~Hz}, 3 \mathrm{H}) ;{ }^{13} \mathrm{C} \mathrm{NMR}(100 \mathrm{MHz}$, $\left.\mathrm{CDCl}_{3}\right) \delta: 161.1(\mathrm{~d}, J=243.7 \mathrm{~Hz}), 140.5,126.0$ (d, $J=8.1$ $\mathrm{Hz}), 114.3$ (d, $J=21.3 \mathrm{~Hz}), 68.8,24.3 ;{ }^{19} \mathrm{~F} \mathrm{NMR}$ (376 MHz, $\left.\mathrm{CDCl}_{3}\right) \delta$ : -115.5 ; Enantiomeric excess was determined by HPLC with a Chiralcel OJ-H column [V(hexane)/ $\left.V\left({ }^{i} \mathrm{PrOH}\right)=70 / 30,1.0 \mathrm{~mL} / \mathrm{min}, 210 \mathrm{~nm}\right]$, minor enantiomer $t_{\mathrm{r}}=23.04 \mathrm{~min}$, major enantiomer $t_{\mathrm{r}}=24.27 \mathrm{~min}$.

(S)-1-(4-Chlorophenyl)ethan-1-ol (10d): Colorless oil, $0.0423 \mathrm{~g}, 90 \%$ yield, $90 \%$ ee. $[\alpha]_{\mathrm{D}}^{27}-35.7\left(\mathrm{c} 1.17, \mathrm{CHCl}_{3}\right)$ $\left[\right.$ lit. $^{[16 \mathrm{c}]}[\alpha]_{\mathrm{D}}^{26}+38.7$ (c 1.12, $\left.\mathrm{CHCl}_{3}\right) \quad(93 \%$ ee for $R$-isomer)]; ${ }^{1} \mathrm{H}$ NMR (400 MHz, $\left.\mathrm{CDCl}_{3}\right) \delta: 7.24 \sim 7.16(\mathrm{~m}$, $4 \mathrm{H}), 4.76(\mathrm{~d}, J=6.4 \mathrm{~Hz}, 1 \mathrm{H}), 2.18$ (brs, $1 \mathrm{H}), 1.36$ (d, $J=6.4$ $\mathrm{Hz}, 3 \mathrm{H}) ;{ }^{13} \mathrm{C}$ NMR $\left(100 \mathrm{MHz}, \mathrm{CDCl}_{3}\right) \delta: 144.3,133.0$, 128.6, 126.8, 69.7, 25.2; Enantiomeric excess was determined by HPLC with a Chiralpak IC-H column $\left[V(\right.$ hexane $\left.) / V\left({ }^{i} \mathrm{PrOH}\right)=99 / 1,1.0 \mathrm{~mL} / \mathrm{min}, 210 \mathrm{~nm}\right]$, major enantiomer $t_{\mathrm{r}}=20.60 \mathrm{~min}$, minor enantiomer $t_{\mathrm{r}}=22.87 \mathrm{~min}$.

(S)-1-(4-Bromophenyl)ethan-1-ol (10e): Colorless oil, $0.0558 \mathrm{~g}, 93 \%$ yield, $87 \%$ ee. $[\alpha]_{\mathrm{D}}^{27}-49.6\left(\mathrm{c} 1.6, \mathrm{CH}_{2} \mathrm{Cl}_{2}\right)$ $\left[\right.$ lit. $^{[16 \mathrm{~d}]}[\alpha]_{\mathrm{D}}^{24}+30.86\left(c \quad 0.915, \mathrm{CHCl}_{3}\right) \quad(95 \%$ ee for $R$-isomer)]; ${ }^{1} \mathrm{H}$ NMR (400 MHz, $\left.\mathrm{CDCl}_{3}\right) \delta: 7.45(\mathrm{~d}, J=8.4$ $\mathrm{Hz}, 2 \mathrm{H}), 7.21$ (d, $J=8.4 \mathrm{~Hz}, 2 \mathrm{H}), 4.82$ (q, $J=6.4 \mathrm{~Hz}, 1 \mathrm{H})$, 2.26 (brs, 1H), 1.44 (d, $J=6.4 \mathrm{~Hz}, 3 \mathrm{H}) ;{ }^{13} \mathrm{C}$ NMR $(100$ $\left.\mathrm{MHz}, \mathrm{CDCl}_{3}\right) \delta: 144.8,131.5,127.2,121.1,69.7,25.2$; Enantiomeric excess was determined by HPLC with a Chiralcel OD-H column $\left[V(\right.$ hexane $) / V\left({ }^{i} \mathrm{PrOH}\right)=98 / 2,1.0$ $\mathrm{mL} / \mathrm{min}, 210 \mathrm{~nm}$, major enantiomer $t_{\mathrm{r}}=17.23 \mathrm{~min}$, minor enantiomer $t_{\mathrm{r}}=19.42 \mathrm{~min}$.

(S)-1-([1,1'-Biphenyl]-4-yl)ethan-1-ol (10f): White solid, m.p. 93 $\sim 95{ }^{\circ} \mathrm{C} ; 0.0567 \mathrm{~g}, 95 \%$ yield, $86 \%$ ee. $[\alpha]_{\mathrm{D}}^{27}-39.3$ $\left(c 1.01, \mathrm{CHCl}_{3}\right)\left[\right.$ lit. $^{[16 \mathrm{e}]}[\alpha]_{\mathrm{D}}^{20}-30.0\left(c 0.94, \mathrm{CHCl}_{3}\right)(70 \%$ $e e$ for $S$-isomer)]; ${ }^{1} \mathrm{H}$ NMR (400 MHz, $\left.\mathrm{CDCl}_{3}\right) \delta: 7.58 \sim$ $7.50(\mathrm{~m}, 4 \mathrm{H}), 7.44 \sim 7.35(\mathrm{~m}, 4 \mathrm{H}), 7.35 \sim 7.28(\mathrm{~m}, 1 \mathrm{H})$, $4.92 \sim 4.81(\mathrm{~m}, 1 \mathrm{H}), 2.40(\mathrm{~s}, 1 \mathrm{H}), 1.48(\mathrm{~d}, J=6.4 \mathrm{~Hz}, 3 \mathrm{H})$; ${ }^{13} \mathrm{C}$ NMR $\left(100 \mathrm{MHz}, \mathrm{CDCl}_{3}\right) \delta: 145.0,140.9,140.4,128.9$, 127.3, 127.3, 127.2, 126.0, 70.1, 25.2; Enantiomeric excess was determined by HPLC with a Chiralpak AD-H column $\left[V(\right.$ hexane $\left.) / V\left({ }^{i} \mathrm{PrOH}\right)=95 / 5,0.8 \mathrm{~mL} / \mathrm{min}, 220 \mathrm{~nm}\right]$, major enantiomer $t_{\mathrm{r}}=35.88 \mathrm{~min}$, minor enantiomer $t_{\mathrm{r}}=41.45 \mathrm{~min}$.

(S)-1-(Benzofuran-2-yl)ethan-1-ol (10g): White solid, m.p. 39 $42{ }^{\circ} \mathrm{C} ; 0.0461 \mathrm{~g}, 94 \%$ yield, 84\% ee. $[\alpha]_{\mathrm{D}}^{27}-12.4$ $\left(c 1.38, \mathrm{CHCl}_{3}\right)\left[\right.$ lit. $^{[16 \mathrm{f}]}[\alpha]_{\mathrm{D}}^{26}+15.9\left(c 0.96, \mathrm{CHCl}_{3}\right)(93 \%$ ee for $R$-isomer)]; ${ }^{1} \mathrm{H}$ NMR $\left(500 \mathrm{MHz}, \mathrm{CDCl}_{3}\right) \delta: 7.49 \sim$ $7.44(\mathrm{~m}, 1 \mathrm{H}), 7.42 \sim 7.38(\mathrm{~m}, 1 \mathrm{H}), 7.24 \sim 7.11(\mathrm{~m}, 2 \mathrm{H}), 6.50$ $(\mathrm{s}, 1 \mathrm{H}), 4.98 \sim 4.87(\mathrm{~m}, 1 \mathrm{H}), 3.08(\mathrm{~s}, 1 \mathrm{H}), 1.55$ (d, $J=6.5$ $\mathrm{Hz}, 3 \mathrm{H}) ;{ }^{13} \mathrm{C}$ NMR $\left(125 \mathrm{MHz}, \mathrm{CDCl}_{3}\right) \delta: 160.4,154.8$, 128.3, 124.2, 122.8, 121.1, 111.3, 101.8, 64.1, 21.5; Enan- 
tiomeric excess was determined by HPLC with a Chiralpak AS-H column $[V$ (hexane $) / V\left({ }^{i} \mathrm{PrOH}\right)=98 / 2,1.0 \mathrm{~mL} / \mathrm{min}$, $254 \mathrm{~nm}$, major enantiomer $t_{\mathrm{r}}=20.60 \mathrm{~min}$, minor enantiomer $t_{\mathrm{r}}=22.87 \mathrm{~min}$.

(S)-1-(Naphthalen-2-yl)ethan-1-ol (10h): White solid, m.p. $71 \sim 73{ }^{\circ} \mathrm{C} ; 0.0490$ g, $95 \%$ yield, $81 \%$ ee. $[\alpha]_{\mathrm{D}}^{27}$ $-42.4\left(c \quad 1.05, \mathrm{CH}_{2} \mathrm{Cl}_{2}\right)$ [lit. ${ }^{[16 \mathrm{~b}]}[\alpha]_{\mathrm{D}}^{20}+37.0$ (c 0.50 , $\left.\mathrm{CH}_{2} \mathrm{Cl}_{2}\right)\left(99 \%\right.$ ee for $R$-isomer)]; ${ }^{1} \mathrm{H} \mathrm{NMR}(400 \mathrm{MHz}$, $\left.\mathrm{CDCl}_{3}\right) \delta: 7.84 \sim 7.75(\mathrm{~m}, 4 \mathrm{H}), 7.73(\mathrm{~s}, 1 \mathrm{H}), 7.49 \sim 7.38(\mathrm{~m}$, $3 \mathrm{H}), 4.98$ (q, $J=6.4 \mathrm{~Hz}, 1 \mathrm{H}), 2.33$ (br s, 1H), 1.52 (d, $J=$ $6.4 \mathrm{~Hz}, 3 \mathrm{H}) ;{ }^{13} \mathrm{C}$ NMR $\left(100 \mathrm{MHz}, \mathrm{CDCl}_{3}\right) \delta: 143.3,133.4$, 133.0, 128.3, 128.0, 127.7, 126.2, 125.8, 123.9, 123.8, 70.5, 25.1; Enantiomeric excess was determined by HPLC with a Chiralcel OD-H column $\left[V(\right.$ hexane $) / V\left({ }^{i} \mathrm{PrOH}\right)=98 / 2,1.0$ $\mathrm{mL} / \mathrm{min}, 210 \mathrm{~nm}$, major enantiomer $t_{\mathrm{r}}=34.64 \mathrm{~min}$, minor enantiomer $t_{\mathrm{r}}=45.86 \mathrm{~min}$.

Supporting Information ${ }^{1} \mathrm{H}$ NMR and ${ }^{13} \mathrm{C}$ NMR spectra of compounds $\mathbf{2 a} \sim \mathbf{2 e}, \mathbf{5}, \mathbf{6}, \mathbf{7 b}, \mathbf{8 b}, \mathbf{1 0 a} \sim \mathbf{1 0 h}$ and $\mathrm{X}$-ray crystal structure and data of $(S)$-2e. Determination of enantiomeric excess of compounds $\mathbf{1 0 a} \sim \mathbf{1 0 h}$. The Supporting Information is available free of charge via the Internet at http://sioc-journal.cn/.

\section{References}

[1] Welch, G. C.; San Juan, R. R.; Masuda, J. D.; Stephan, D. W. Science 2006, 314, 1124.

[2] (a) Stephan, D. W. Org. Biomol. Chem. 2008, 6, 1535.

(b) Kenward, A. L.; Piers, W. E. Angew. Chem., Int. Ed. 2008, 47, 38.

(c) Stephan, D. W.; Erker, G. Angew. Chem., Int. Ed. 2010, 49, 46.

(d) Soós, T. Pure Appl. Chem. 2011, 83, 667.

(e) Erker, G. Pure Appl. Chem. 2012, 84, 2203.

(f) Stephan, D. W. Org. Biomol. Chem. 2012, 10, 5740

(g) Paradies, J. Angew. Chem., Int. Ed. 2014, 53, 3552.

(h) Stephan, D. W. Acc. Chem. Res. 2015, 48, 306.

(i) Stephan, D. W.; Erker, G. Angew. Chem., Int. Ed. 2015, 54, 6400.

(j) Oestreich, M.; Hermeke, J.; Mohr, J. Chem. Soc. Rev. 2015, 44, 2202.

(k) Stephan, D. W. Science 2016, 354, aaf7229.

[3] (a) Liu, Y.; Du, H. Acta Chim. Sinica 2014, 72, 771 (in Chinese). (刘勇兵, 杜海峰, 化学学报, 2014, 72, 771.)

(b) Feng, X.; Du, H. Tetrahedron Lett. 2014, 55, 6959.

(c) Shi, L.; Zhou, Y.-G. ChemCatChem 2015, 7, 54.

(d) Meng, W.; Feng, X.; Du, H. Acc. Chem. Res. 2018, 51, 191.

[4] (a) Sumerin, V.; Chernichenko, K.; Nieger, M.; Leskelä, M.; Rieger, B.; Repo, T. Adv. Synth. Catal. 2011, 353, 2093.

(b) Mewald, M.; Fröhlich, R.; Oestreich, M. Chem.-Eur. J. 2011, 17, 9406 .

(c) Mewald, M.; Oestreich, M. Chem.-Eur. J. 2012, 18, 14079.

(d) Lindqvist, M.; Borre, K.; Axenov, K.; Kótai, B.; Nieger, M.; Leskelä, M.; Pápai, I.; Repo, T. J. Am. Chem. Soc. 2015, 137, 4038. (e) Süsse, L.; Hermeke, J.; Oestreich, M. J. Am. Chem. Soc. 2016,
138,6940 .

(f) Lam, J.; Günther, B. A. R.; Farrell, J. M.; Eisenberger, P.; Bestvater, B. P.; Newman, P. D.; Melen, R. L.; Crudden, C. M.; Stephan, D. W. Dalton Trans. 2016, 45, 15303.

[5] (a) Chen, D.; Wang, Y.; Klankermayer, J. Angew. Chem., Int. Ed. 2010, 49, 9475 .

(b) Chen, D.; Leich, V.; Pan, F.; Klankermayer, J. Chem. - Eur. J. 2012, 18, 5184 .

(c) Ghattas, G.; Chen, D.; Pan, F.; Klankermayer, J. Dalton Trans. 2012, 41,9026 .

(d) Ye, K.-Y.; Wang, X.; Daniliuc, C. G.; Kehr, G.; Erker, G. Eur. J. Inorg. Chem. 2017, 368.

(e) Chen, D.; Klankermayer, J. Chem. Commun. 2008, 2130.

[6] (a) Parks, D. J.; Spence, R. E. von H.; Piers, W. E. Angew. Chem., Int. Ed. 1995, 34, 809.

(b) Parks, D. J.; Piers, W. E.; Yap, G. P. A. Organometallics 1998, $17,5492$.

[7] (a) Liu, Y.; Du, H. J. Am. Chem. Soc. 2013, 135, 12968.

(b) Wei, S.; Du, H. J. Am. Chem. Soc. 2014, 136, 12261.

(c) Zhang, Z.; Du, H. Angew. Chem., Int. Ed. 2015, 54, 623.

(d) Ren, X.; Du, H. J. Am. Chem. Soc. 2016, 138, 810.

(e) Liu, X.; Wang, Q.; Han, C.; Feng, X.; Du, H. Chin. J. Chem. 2019, 37, 663 .

[8] (a) Tu, X.-S.; Zeng, N.-N.; Li, R.-Y.; Zhao, Y.-Q.; Xie, D.-Z.; Peng Q.; Wang, X.-C. Angew. Chem., Int. Ed. 2018, 57, 15096.

(b) Li, X.; Tian, J.-J.; Liu, N.; Tu, X.-S.; Zeng, N.-N.; Wang, X.-C. Angew. Chem., Int. Ed. 2019, 58, 4664.

[9] Zhou, Q.-L., Privileged Chiral Ligands and Catalysts, Wiley-VCH, Weinheim, Germany, 2011.

[10] For selected reviews, see: (a) Xie, J.-H.; Zhou, Q.-L. Acc. Chem. Res. 2008, $41,581$.

(b) Ding, K.-L.; Han, Z.-B.; Wang, Z. Chem. Asian J. 2009, 4, 32.

(c) Xie, J.-H.; Zhou, Q.-L. Acta Chim. Sinica 2014, 72, 778 (in Chinese).

(谢建华, 周其林, 化学学报, 2014, 72, 778.)

(d) Xie, J.-H.; Bao, D.-H.; Zhou, Q.-L. Synthesis 2015, 47, 460.

(d) Zhu, S.-F.; Zhou, Q.-L. Acc. Chem. Res. 2012, 45, 1365.

[11] For a pioneering work, see: Parks, D. J.; Piers, W. E. J. Am. Chem. Soc. 1996, $118,9440$.

[12] Rendler, S.; Oestreich, M. Angew. Chem., Int. Ed. 2008, 47, 5997.

[13] Zhu, S.-F.; Yang, Y.; Wang, L.-X.; Liu, B.; Zhou, Q.-L. Org. Lett. 2005, 7, 2333.

[14] Zheng, J.; Cui, W.-J.; Zheng, C.; You, S.-L. J. Am. Chem. Soc. 2016, 138,5242 .

[15] For details, see the Supporting Information. CCDC 1905630 contains the supplementary crystallographic data for this paper. These data can be obtained free of charge from The Cambridge Crystallographic Data Centre via www.ccdc.cam.ac.uk/data_request/cif.

[16] (a) Sokeirik, Y. S.; Mori, H.; Omote, M; Sato, K.; Tarui, A.; Kumadaki, I.; Ando, A. Org. Lett. 2007, 9, 1927.

(b) Wu, W.; Liu, S.; Duan, M.; Tan, X.; Chen, C.; Xie, Y.; Lan, Y.; Dong, X.; Zhang, X. Org. Lett. 2016, 18, 2938.

(c) Ren, X.; Li, G.; Wei, S.; Du, H. Org. Lett. 2015, 17, 990.

(d) Zhang, Z.; Jain, P.; Antilla, J. C. Angew. Chem., Int. Ed. 2011, 50, 10961

(e) Süsse, L.; Hermeke, J.; Oestreich, M. J. Am. Chem. Soc. 2016, 138,6940 .

(f) Chen, X.; Lu, Z. Org. Lett. 2016, 18, 4658. 